Research Article

\title{
Multiscale Hedging with Crude Oil Futures Based on EMD Method
}

\author{
Chengli Zheng $\mathbb{D}^{1,2}$ and Kuangxi Su $\mathbb{D}^{1,2}$ \\ ${ }^{1}$ School of Economics and Business Administration, Central China Normal University, Wuhan, China \\ ${ }^{2}$ Financial Engineering Research Center, Central China Normal University, Wuhan, China \\ Correspondence should be addressed to Kuangxi Su; k1152010@163.com
}

Received 29 August 2020; Revised 8 October 2020; Accepted 20 October 2020; Published 2 November 2020

Academic Editor: Bekir Sahin

Copyright (c) 2020 Chengli Zheng and Kuangxi Su. This is an open access article distributed under the Creative Commons Attribution License, which permits unrestricted use, distribution, and reproduction in any medium, provided the original work is properly cited.

\begin{abstract}
Studying the impact of the different components in data on hedging can provide valuable guidance to investors. However, the previous multiscale hedging studies do not examine the issue from the data itself. In this study, we use the empirical mode decomposition (EMD) method to reconstruct the crude oil futures and spot returns into three different scales: short-term, medium-term, and long-term. Then, we discuss the crude oil hedging performance under the dynamic minimum-CVaR framework at different scales. Based on the daily prices of Brent crude oil futures contract from August 18, 2005, to September 16, 2019, the empirical results show that the extracted scales comprise different information of original returns, short-term information occupies the most important position, and hedging is mainly driven by short-term information. Besides, hedging relying on long-term information has the best hedging performance. Removing some information related to short-term noise from the original returns is helpful for investors.
\end{abstract}

\section{Introduction}

As "the blood of industry," crude oil has become an important energy source that can affect economic activity and financial markets [1-3]; over the last decades, numerous market participants chose crude oil futures to avoid adverse spot price fluctuations [4]. Since the futures market consists of many participants with different trading cycles, the resulting time series comprise different time and frequency domain components [5]; studying the impact of these different components on hedging can provide valuable guidance to investors. However, the previous multiscale hedging studies ignore this important issue; in this paper, we divide the data into short-, medium-, and long-term scales and explore the impact of different scales on hedging.

In practice, one of the main theoretical issues in hedging involves the determination of the optimal hedge ratio. The extant studies obtain optimal hedge ratios from the perspective of improving the objective function. [6, 7]. For example, the classical minimum-variance (MV) hedging uses variance as a risk proxy [8-10]. However, variance is not a perfect measure because it treats upside potential and downside risk equally in risk management and investors pay more attention to downside risk compared to upside risk. Thus, several downside risk measures have been introduced in hedging practice, such as Value-at-Risk (VaR) [11] and Conditional Value-at-Risk (CVaR) [12]. The criticism against $\mathrm{VaR}$ originates from its lack of subadditivity and convexity [13]. Besides, $\mathrm{VaR}$ is not easy to optimize when calculated using scenarios [14]. For these reasons, CVaR, which can be thought of as a coherent risk measure, has been widely applied in the hedging field. Naturally, the impact of different scales on hedging is mainly discussed under the minimum-CVaR framework.

Another important issue focusing on hedging is the dependence of the optimal hedge ratio on the data with different frequencies. As argued by Chun et al. [15], the frequency of data used to estimate hedge ratio is important, and any incompatible frequencies could result in incorrect hedge ratios, in turn impacting hedging performance and 
accuracy. Some studies have begun to take note of this problem; for example, Colon et al. [16] use wavelet analysis to compute the hedge ratios at different time scales; they find that the hedging effectiveness is significantly improved with scale increasing. Susan et al. [17] also compare different models at different scales from the aspects of portfolio variance and utility function; their results indicate that the wavelet networks offer improvements over traditional hedging models. These studies focus on the hedging effectiveness from the perspective of hedging horizon. That is, investors may use the daily, weekly, monthly, or quarterly data to estimate the optimal hedge ratio. However, the impact of different scales on hedging is ignored; observing hedging from the perspective of the data itself usually provides more comprehensive information.

Except for the popular wavelet decomposition method [18], empirical mode decomposition (EMD) also received extensive attention [19]. EMD can directly decompose original data into finite intrinsic mode functions (IMFs) and a trend item without prior assumptions about signal modes or system orders, which has shown outstanding advantages in nonlinear and nonstationary signals analysis. At present, EMD has been widely applied to decompose financial data [20-22]. Based on these superior properties of EMD, we use EMD to decompose futures and spot returns and further construct three different scales. To the best of our knowledge, this is the first study to apply the decomposition method to multiscale hedging.

In this paper, we consider multiscale hedging in view of the data itself. In detail, the EMD technology is first introduced to decompose crude oil futures and spot returns; the decomposition terms are reconstructed into three different scales: short-term, medium-term, and long-term. Then, we discuss the scale dependency structure through three key indicators: variance contribution rate, Shapley value, and correlation coefficient. Lastly, under the minimum-CVaR framework, two different hedging strategies, constant hedging strategy and dynamic hedging strategy using DCC-GARCH model, are selected to comprehensively analyze the crude oil hedging performance at different scales.

In the empirical analysis, the daily closing prices of spot and futures contracts of Brent crude oil were from August 18, 2005, to September 16, 2019, and a total of 3379 price observations were collected to test in-sample and out-of-sample hedging effectiveness. We select the CVaR, variance, returns, and utility as the criteria to provide an in-depth and comprehensive assessment of the hedging performance at different scales. The empirical results show that the extracted scales comprise different information of original returns, Secondly, short-term information occupies the most important position, and hedging is mainly driven by short-term information. Lastly, hedging relying on long-term information has the best hedging performance; excluding information related to short-term noise from the original returns is helpful for investors. Robustness results by changing the objective function to variance validate the above conclusions. These findings are distinctly different from the previous literature.
The remainder of this paper is organized as follows: Section 2 briefly introduces the methodology, including the EMD technique, two downside risk measures, and the hedging theory. Section 3 introduces the data sources. The empirical results are reported in Section 4, which includes the multiscale analysis and hedging results. The last section concludes the paper.

\section{Model and Methodology}

In order to comprehensively analyze the hedging performance at different scales, we used the EMD technique to decompose the crude oil spot and futures returns and grouped the decomposition terms into three different scales: short-term, medium-term, and long-term. Then, the hedging performance is comprehensively assessed under the minimum-CVaR framework. Figure 1 shows the basic framework of this paper.

2.1. Empirical Mode Decomposition (EMD). The empirical mode decomposition was originally proposed by Huang et al. [19]. The EMD decomposes the original data into a series of intrinsic mode functions (IMFs), and the IMFs need to satisfy the two following conditions: First, in the whole time series, the extremum numbers and zero-crossing points must be equal or differ at most by one. Second, at any point, the mean value of the envelope defined by the local maxima and the envelope defined by the local minima is zero. With this definition, any complicated data series $x_{t}(t=1,2, \ldots, T)$ can be decomposed as follows:

(1) Identify the local extrema of $x_{t}$, including both maxima and minima

(2) Calculate its upper and lower envelopes, $x_{\mathrm{up}, t}$ and $x_{\text {low }, t}$, with cubic spline interpolation

(3) Compute the point-by-point means $m_{i}$ from upper and lower envelopes: $m_{t}=\left(x_{\text {up }, t}+x_{\text {low }, t}\right) / 2$

(4) Extract the means from the time series to obtain an IMF candidate $c_{t}=x_{t}-m_{t}$

(5) Check the properties of $c_{t}$ : If $c_{t}$ meets the above two conditions, then IMF is extracted and $x_{t}$ is replaced with the residue $r_{t}=x_{t}-c_{t}$. If $c_{t}$ is not an IMF, $x_{t}$ is replaced with $c_{t}$

(6) Repeat steps 1-5 until the stop criterion is satisfied and no more IMFs can be extracted

Using this sifting procedure, the original data series $x_{t}$ can finally be expressed as the sum of IMFs and a residual:

$$
x_{t}=\sum_{j=1}^{k} c_{j, t}+r_{t}
$$

where $k$ is the number of IMFs, $r_{t}$ is the residual, and $c_{j, t}(j=1,2, \ldots, k)$ is the $j^{\text {th }}$ IMF.

2.2. Downside Risk Measures. As the crude oil trading increases in trading volume and liquidity, investors are increasingly paying attention to downside risk. However, 


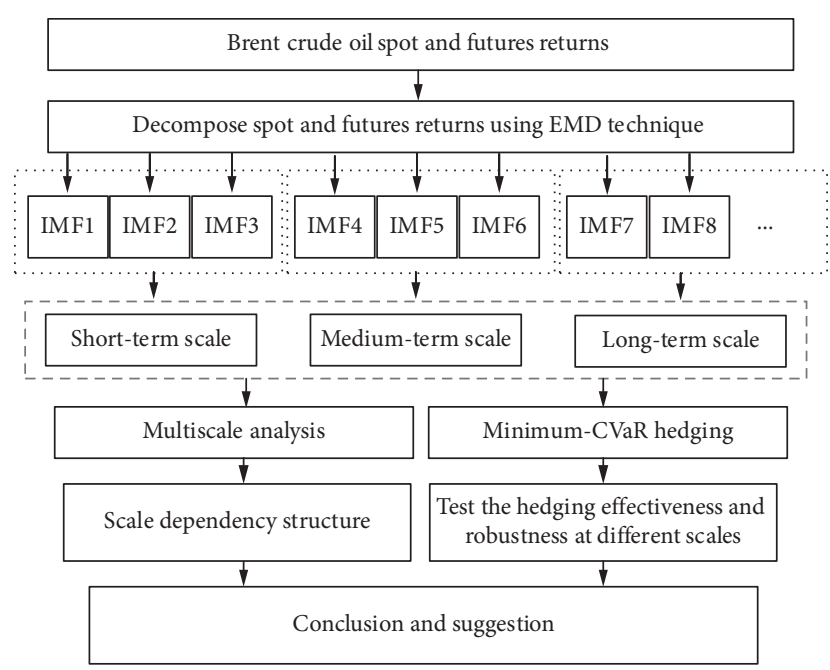

Figure 1: The basic framework of this paper.

variance treats upside potential and downside risk equally in risk management. As a downside risk measure, VaR focuses on the probability not the magnitude of risk. Generally, the $1-\alpha$ percent $\mathrm{VaR}$ of portfolio returns $p$ is expressed as

$$
\operatorname{VaR}_{p}(1-\alpha)=-\inf \{u: F(u)>1-\alpha\}
$$

where $F$ is the cumulative distribution function (CDF) of portfolio returns $p$.

As $\mathrm{VaR}$ lacks theoretical property for coherent risk measures, an alternative risk measure that addresses this shortcoming is $\mathrm{CVaR}$, which is defined as the conditional expectation of losses exceeding $\mathrm{VaR}$ for continuous distributions.

$$
\begin{aligned}
\operatorname{CVaR}_{p}(1-\alpha) & =-E\left[R_{p} \mid R_{p} \leq-\operatorname{VaR}_{p}(1-\alpha)\right] \\
& =\frac{1}{\alpha} \int_{1-\alpha}^{1} \operatorname{VaR}_{p}(x) \mathrm{d} x .
\end{aligned}
$$

2.3. Semiparametric Estimation for $V a R / C V a R$. As the evidence shows that the features with the heavy-tailed and higher kurtosis distribution exist in financial market, we use an analytical expression for VaR derived from the CornishFisher expansion [23], which approximates the quantile of a standardized probability distribution using the higher moments of the distribution. The Cornish-Fisher expansion approximates $c(\alpha)$ by

$$
\begin{aligned}
\widetilde{c}_{p}\left(\alpha ; s_{p}, k_{p}\right) \approx & c(\alpha)+\frac{1}{6}\left[c(\alpha)^{2}-1\right] s_{p} \\
& +\frac{1}{24}\left[c(\alpha)^{3}-3 c(\alpha)\right]\left(k_{p}-3\right) \\
& -\frac{1}{36}\left[2 c(\alpha)^{3}-5 c(\alpha)\right] s_{p}^{2},
\end{aligned}
$$

where $c(\alpha)$ is the $\alpha$ percent quantile of the standard normal distribution and $s_{p}$ and $k_{p}$ are the skewness and kurtosis of the portfolio returns $p$, respectively. The Cornish-Fisher approximations for $\mathrm{VaR}$ and $\mathrm{CVaR}$ are then expressed as

$$
\begin{aligned}
\operatorname{VaR}_{p}(1-\alpha)= & -\sigma_{p} \tilde{c}_{p}\left(1-\alpha ; s_{p}, k_{p}\right)-\mu_{p}, \\
\mathrm{CVaR}_{p}(1-\alpha)= & -\sigma_{p}\left[M_{1}+\frac{1}{6}\left(M_{2}-1\right) s_{p}\right. \\
& +\frac{1}{24}\left(M_{3}-3 M_{1}\right)\left(k_{p}-3\right) \\
& \left.-\frac{1}{36}\left(2 M_{3}-5 M_{1}\right) s_{p}^{2}\right]-\mu_{p},
\end{aligned}
$$

where $M_{i}=1 /(1-\alpha) \epsilon_{-\infty}^{c(1-\alpha)} x^{i} f(x) \mathrm{d} x, \quad i=1,2,3, \ldots$, and $f($.) is the standard normal probability density function.

2.4. Minimum-CVaR Hedging. Hedging is the main function of futures markets. The key procedure in futures hedging is to compute the optimal hedge ratio. Consider a portfolio consisting of $C_{S}$ units of a long spot position and $C_{F}$ units of a short futures position. Let $S_{t}$ and $F_{t}$ denote the natural logarithms of spot and futures prices at the end of period $t$, respectively. The return on the hedged portfolio over a period, $\Delta P_{t}$, is given by

$$
\Delta P_{t}=C_{s} \Delta S_{t}-C_{f} \Delta F_{t},
$$

where $\Delta S_{t}=S_{t}-S_{t-1}$ and $\Delta F_{t}=F_{t}-F_{t-1}$.

The optimal hedge ratio is derived by minimizing the conditional variance of $\Delta P_{t}$. Thus, it is also called the minimum-variance hedge ratio and is given by

$$
h_{t}^{*}=\frac{C_{f}}{C_{s}}=\frac{\operatorname{Cov}\left(\Delta S_{t}, \Delta F_{t} \mid \Omega_{t}\right)}{\operatorname{Var}\left(\Delta F_{t} \mid \Omega_{t}\right)},
$$

where $\Omega_{t}$ is the appropriate information set.

However, some scholars [12, 23] argue that variance is not a good measure of portfolio risk. As an alternative, the hedge ratios of minimum- $\mathrm{VaR} / \mathrm{CV}$ aR are calculated as 


$$
\begin{aligned}
\frac{\partial V a R_{p}}{\partial h_{t}}= & \frac{\partial \sigma_{p t}}{\partial h_{t}}\left(A_{1}+A_{2} s_{p}+A_{3} k_{p}+A_{4} s_{p}^{2}\right) \\
& +\sigma_{p t}\left(A_{2} \frac{\partial s_{p}}{\partial h_{t}}+A_{3} \frac{\partial k_{p}}{\partial h_{t}}+2 A_{4} s_{p} \frac{\partial s_{p}}{\partial h_{t}}\right)+\mu_{f t}=0
\end{aligned}
$$

$$
\begin{aligned}
\frac{\partial C V a R_{p}}{\partial h_{t}}= & \frac{\partial \sigma_{p t}}{\partial h_{t}}\left(B_{1}+B_{2} s_{p}+B_{3} k_{p}+B_{4} s_{p}^{2}\right) \\
& +\sigma_{p t}\left(B_{2} \frac{\partial s_{p}}{\partial h_{t}}+B_{1} \frac{\partial k_{p}}{\partial h_{t}}+2 B_{4} s_{p} \frac{\partial s_{p}}{\partial h_{t}}\right)+\mu_{f t}=0,
\end{aligned}
$$

where $\mu_{f t}$ is the conditional mean of futures, IbeginAlign

$$
\begin{aligned}
& A_{1}=c(\alpha)-\frac{1}{8}\left[c(\alpha)^{3}-3 c(\alpha)\right], \\
& A_{2}=\frac{1}{6}\left[c(\alpha)^{2}-1\right], \\
& A_{3}=\frac{1}{24}\left[c(\alpha)^{3}-3 c(\alpha)\right], \\
& A_{4}=-\frac{1}{36}\left[2 c(\alpha)^{3}-5 c(\alpha)\right] .
\end{aligned}
$$

and

$$
\begin{aligned}
& B_{1}=M_{1}-\frac{1}{8}\left[M_{3}-3 M_{1}\right], \\
& B_{2}=\frac{1}{6}\left[M_{2}-1\right], \\
& B_{3}=\frac{1}{24}\left[M_{3}-3 M_{1}\right], \\
& B_{4}=-\frac{1}{36}\left[2 M_{3}-5 M_{1}\right] .
\end{aligned}
$$

lendAlign

Besides, some scholars argue that the hedging model varies over time because the factors that affect hedging performance change over time [7]. To further confirm the credibility of our results, two hedging strategies are considered to estimate the hedge ratios: one is a constant hedging model without complicated technology and the other is using the DCC-GARCH model to get the timevarying hedge ratio. In general, the model can be expressed as follows:

$$
\begin{aligned}
\Delta S_{t} & =a_{0}+a_{1} u_{t-1}+\varepsilon_{s}, \Delta F_{t} \\
& =b_{0}+b_{1} u_{t-1}+\varepsilon_{f}\left(\begin{array}{c}
\varepsilon_{s} \\
\varepsilon_{f}
\end{array}\right) \mid \Omega_{t-1} \sim N\left(0, H_{t}\right),
\end{aligned}
$$

where $H_{t}$ is the conditional covariance matrix.

To capture the time-variation property in matrix $H_{t}$, Engle [24] develops a DCC model, which can be written as

$$
H_{t}=G_{t} \Gamma G_{t}=\left(\rho_{s f, t} \sqrt{h_{s, t} h_{f, t}}\right),
$$

where $\Gamma_{t}=\operatorname{diag}\left(q_{s t}^{-1 / 2}, q_{f t}^{-1 / 2}\right) Q_{t} \operatorname{diag}\left(q_{s, t}^{-1 / 2}, q_{f t}^{-1 / 2}\right)$, and the process of positive definite matrix $Q_{t}$ is given by

$$
Q_{t}=\bar{\rho}\left(1-\lambda_{1}-\lambda_{2}\right)+\lambda_{1}\left(\varepsilon_{t-1} \varepsilon_{t-1}^{\prime}\right)+\lambda_{2} \Gamma_{t-1},
$$

where $\bar{\rho}$ is the unconditional correlation matrix of $\varepsilon_{t}$. The parameters $\lambda_{1}$ and $\lambda_{2}$ are the scalars and satisfy the condition $\lambda_{1}+\lambda_{2}<1$.

\section{Data Resource}

We collect the spot and futures data of Brent crude oil traded under the Intercontinental Exchange (ICE); the Brent crude oil contract is used since it is the major futures trading contract in crude oil market. Besides, due to the high trading liquidity and volume, it can effectively reflect the evolution of energy markets and has been widely used in the hedging literature. The dataset comprises the daily closing prices of 3379 trading days from August 18, 2005, to September 16, 2019. All price data are collected from the wind database.

Figure 2 plots the prices and returns of crude oil spot and futures. We can see that oil price fluctuates fiercely. Some occasional events such as the 2008 financial crisis and the 2015 surge in US oil production can cause crashes in oil prices. Besides, compared with the characteristics of crude oil spot time series, the crude oil futures exhibit the consistent price trend and similar volatility features in Figure 2. This phenomenon appears due to the fact that investors share some common information in these relative markets. As these two markets have similar trends by the same economic factors, operating on them reversely can hedge the risk. The higher the correlation level is, the better the effect of hedging will be. Besides, the tendency for return volatility appears in bunches; large returns are expected to follow large returns, and small returns are expected to follow small returns, due to clustering of information arrivals. This is the effect named volatility clustering.

\section{Empirical Analysis}

In this section, we first discuss the scale dependency structure through three key indicators: variance contribution rate, Shapley value, and correlation coefficient. Then, under the minimum-CVaR framework, two different hedging strategies, the constant hedging strategy and dynamic hedging strategy, are selected to comprehensively analyze the crude oil hedging performance at different scales.

4.1. Multiscale Analysis. After obtaining the data, the EMD method is used to decompose crude oil futures and spot returns. Figure 3 shows the decomposition results for both futures (left panel) and spot (right panel) (Figures 3(a) and $3(b))$ returns. The EMD splits the original time series data into a series of IMFs. Their cycles are from short to long; likewise, their frequencies are from high to low. Besides, the returns of decomposed brent spot exhibit similar patterns (right panel) (Figure 3(b)). 

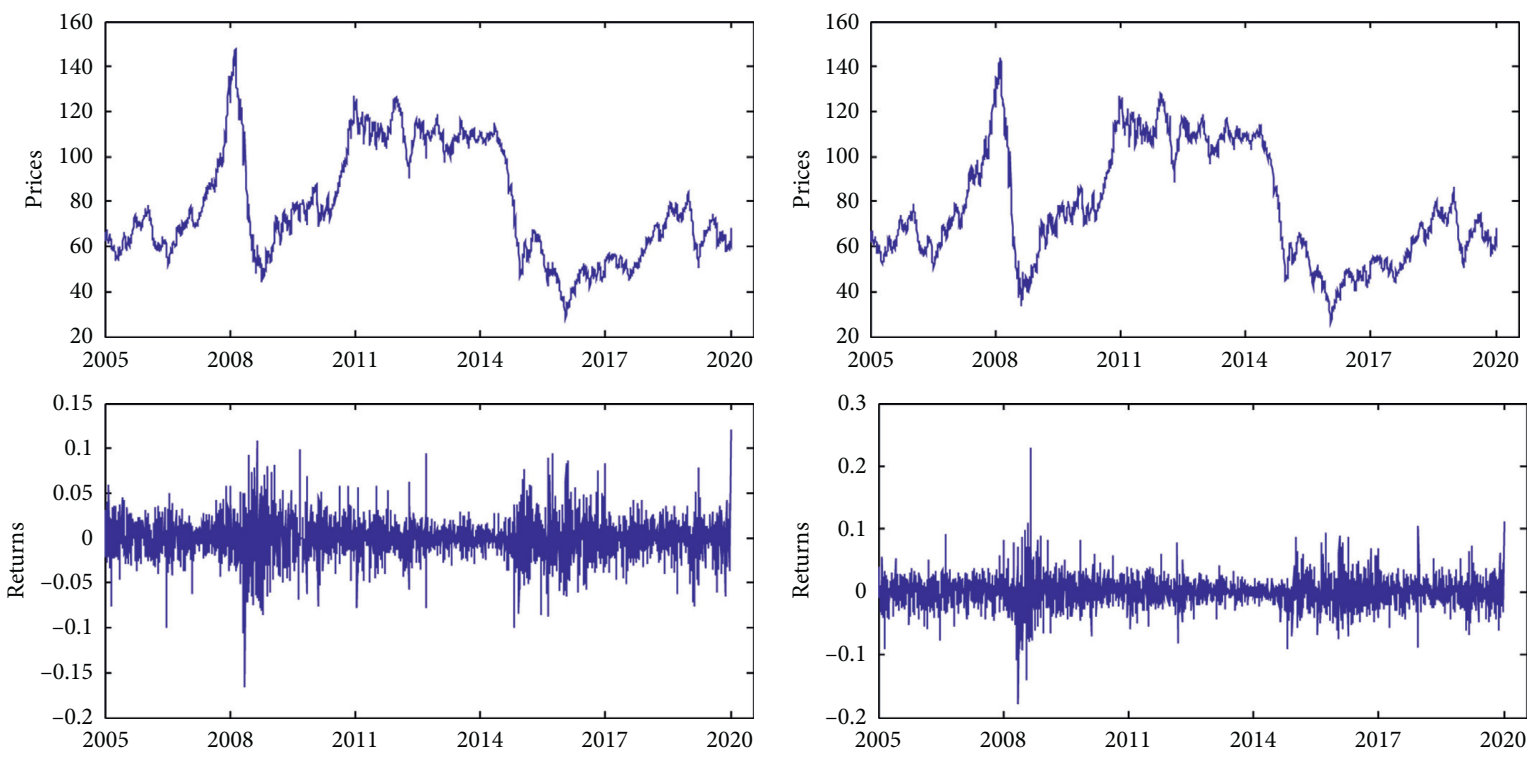

(a)

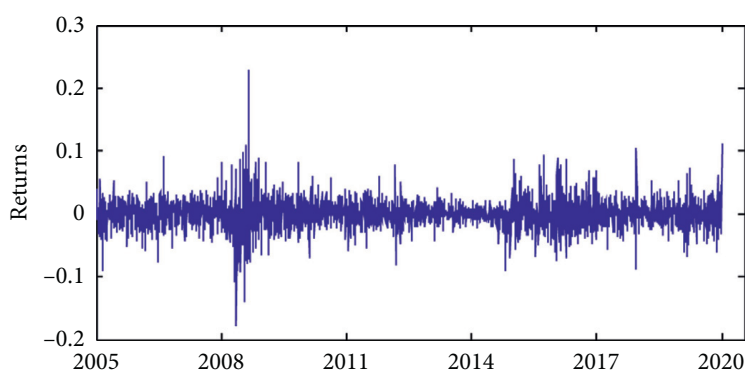

(b)

Figure 2: The characteristics of futures (left) (a) and spot (right) (b).

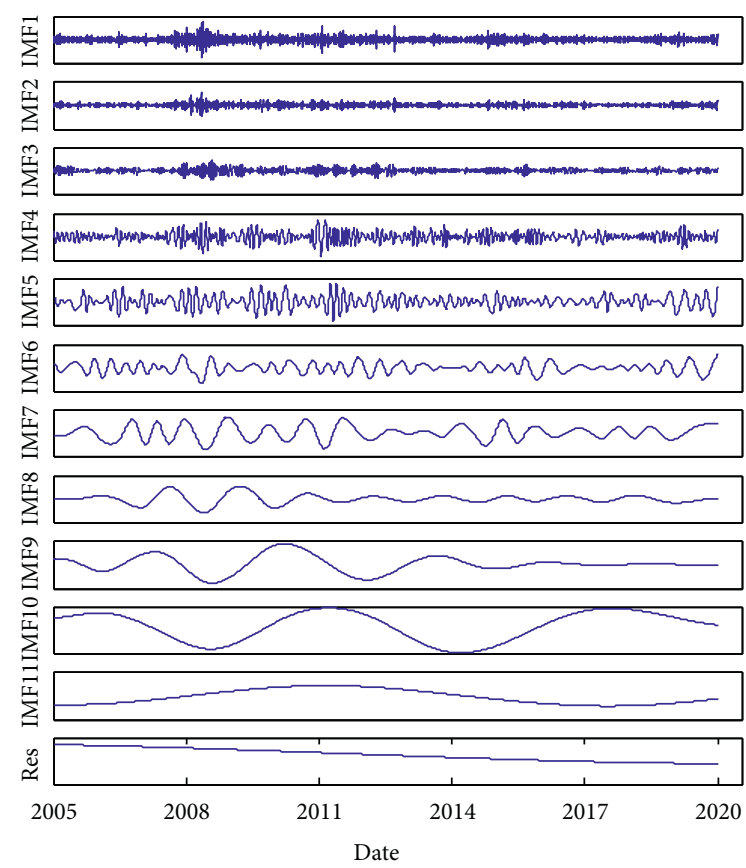

(a)

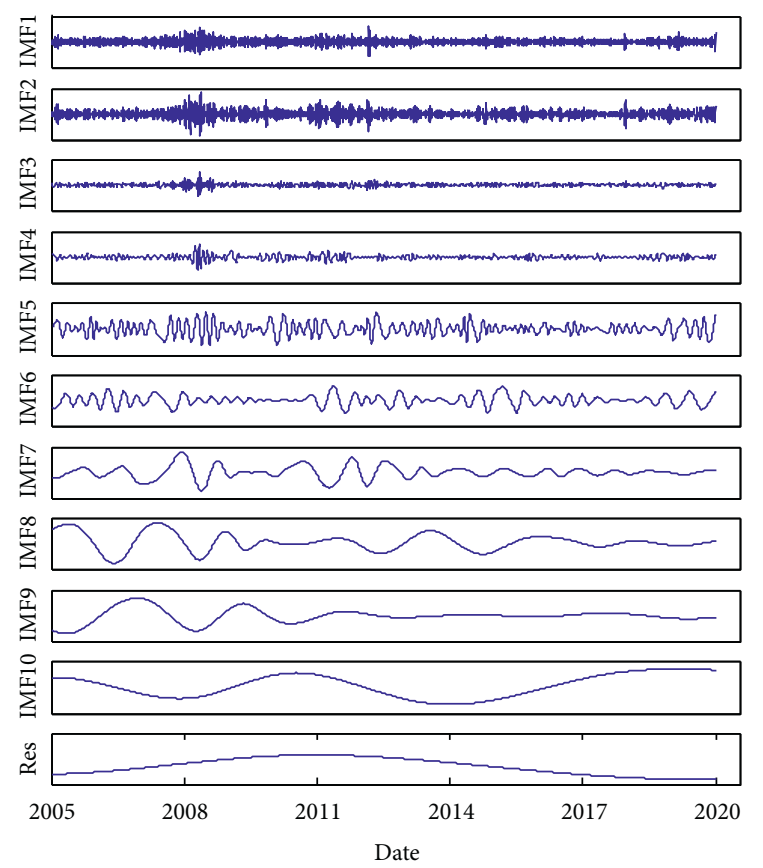

(b)

FIgURE 3: The decomposition terms of futures (left) (a) and spot (right) (b) generated by EMD.

4.1.1. Descriptive Statistics for Different Scales. Since the adjacent IMFs have similar properties, we follow the previous construction method [25] and group the IMFs from high frequency to low frequency into short-term, mediumterm, and long-term time scales. The sum of first three IMFs is called the short-term scale (abbreviated short scale), the sum of the fourth to sixth IMFs is defined as medium-term scale (medium scale), and the sum of the remaining decomposition term is the long-term scale (long scale). The descriptive statistics for each scale are reported in Table 1. We can see that each pair of scales for the two returns series has a similar feature, which indicates the rationality of multiscale hedging theory.

Firstly, each pair of scales has a similar duration and the difference between different scales is very obvious, indicating completely different economic implications. In particular, short scale, the average duration of which is $2-4$ days, is identified as the markets' normal fluctuation, and it happens at a high 
TABle 1: Descriptive statistics of different scales for the futures and spot returns.

\begin{tabular}{|c|c|c|c|c|c|c|c|c|}
\hline & Scale & Mean & $\mathrm{Sd}$ & Skewness & Kurtosis & Jarque-Bera & $Q^{2}(10)$ & Duration (day) \\
\hline \multirow{4}{*}{ Futures } & Original & 0.0017 & 1.5208 & -0.4694 & 11.1475 & $9467.20^{* * *}$ & 369.26 & \\
\hline & Short & 0.0257 & 1.4485 & -0.1692 & 8.9098 & $4931.84^{* * *}$ & $665.65^{* * *}$ & 2.96 \\
\hline & Medium & -0.0019 & 0.5183 & -0.2795 & 3.9026 & $158.66^{* * *}$ & $7856.96^{* * *}$ & 21.11 \\
\hline & Long & -0.0221 & 0.2209 & -0.9265 & 6.3240 & $2038.38^{* * *}$ & $32925.20^{* * *}$ & 198.71 \\
\hline \multirow{4}{*}{ Spot } & Original & 0.0019 & 1.5409 & -0.2962 & 10.9829 & $9018.84^{* * *}$ & $302.66^{* * *}$ & \\
\hline & Short & -0.0068 & 1.4683 & -0.0961 & 8.7481 & $4655.61^{* * *}$ & $384.63^{* * *}$ & 2.96 \\
\hline & Medium & 0.0082 & 0.5300 & -0.0381 & 4.8758 & $496.07^{* * *}$ & $8992.21^{* * *}$ & 22.67 \\
\hline & Long & 0.0005 & 0.2988 & -1.2811 & 7.8007 & $4167.79^{* * *}$ & $32438.47^{* * *}$ & 211.13 \\
\hline
\end{tabular}

"Original" denotes original data. The null hypothesis of Jarque-Bera statistic is the normal distribution. The asterisks ${ }^{*},{ }^{* *}$, and ${ }^{* * *}$ denote rejection of the null hypothesis at $10 \%, 5 \%$, and $1 \%$ significance levels, respectively. The duration represents the mean period of different scales.

TABLE 2: Variance contribution rate and Shapley value of different scales.

\begin{tabular}{lcccccc}
\hline & \multicolumn{3}{c}{ Futures } & \multicolumn{3}{c}{ Spot } \\
& Short & Medium & Long & Short & Medium & Long \\
\hline Variance & 2.0416 & 0.2269 & 0.0442 & 1.2733 & 0.1939 & 0.0637 \\
\% $^{\mathrm{a}}$ & $88.28 \%$ & $9.81 \%$ & $1.91 \%$ & $83.18 \%$ & $12.66 \%$ & $4.16 \%$ \\
Shapley $^{\mathrm{b}}$ & $61.77 \%$ & $26.27 \%$ & $11.96 \%$ & $64.18 \%$ & $27.45 \%$ & $8.37 \%$ \\
\hline
\end{tabular}

${ }^{\mathrm{a}} \%$ denotes variance contribution rate; it is calculated based on the proportion of the variance of different scale and original returns. ${ }^{\mathrm{b}}$ The definition of Shapley value is $v_{i}=\sum_{k=0}^{n-1} \gamma_{k} \sum_{K \subset X \backslash x_{i}}\left(\mu_{i K}-\mu_{K}\right)$, where $\gamma_{k}=(n-k-1) ! k ! / n !=1 / n\left(\begin{array}{l}k \\ 2\end{array}\right) ; \mu$ denotes risk measure; $K$ is power.

frequency. It is mainly caused by a series of factors that have short-term effects such as short-term supply and demand imbalance. Medium scale, the average duration of which is about one month, is mainly caused by factors that have medium-term effects. It includes the effects of minor irregular events and some significant events such as natural disasters like hurricanes and so forth. The long scale has a little tendency towards variation and is smoother compared with the short and medium scales. Its duration is close to one year, which reflects the long-term trend of the markets; it is affected by some longterm events such as the financial crisis and trade war.

Table 2 also reports other descriptive statistics for each scale. We can see that the changes for the two returns are similar, and the means are all close to zero for all scales. Besides, there are lower skewness and standard deviation at a higher scale. What is interesting is the fact that the skewness and kurtosis of spot and futures returns change dramatically at the long scale, mainly caused by the existence of trend term. Meanwhile, all their kurtoses are higher than 3, implying that the returns follow a fattailed distribution. The nonnormality of distribution is also confirmed by the statistical rejections of the null hypothesis based on the Jarque-Bera test. Finally, we use the $Q^{2}$-statistics (Ljung-Box statistics) to test for ARCH effects and find that they are significant for decomposed spot and futures returns; both the original returns and different scales have $\mathrm{ARCH}$ properties. Therefore, the use of the DCC-GARCH model is appropriate.

4.1.2. Dependency Structure. To further analyze the characteristics and importance of different scales, we use the variance contribution rate, Shapley value [26], and correlation coefficient to describe the fluctuation characteristics, global importance, and trends of different scales.

The variance contribution rate can fully extract the fluctuation information of original data and reflect the fluctuation characteristics of different scales. The relevant results are shown in Table 2; we can see that short scale, which is identified as the markets' normal fluctuation $(88.28 \%$ and $83.18 \%$ for the futures and spot, respectively), accounts for the largest share of overall market fluctuations. Meanwhile, the variance of medium scale, which represents fluctuations caused by minor irregular and significant events, is also relatively large, indicating that the shock of minor irregular and significant events can cause large fluctuations. The long scale representing the long-term trend of market contributes minimally to fluctuations, indicating the long-term evolutionary characteristic of crude oil market.

We also introduce the Shapley value to identify the "contribution" or "weight" of different scales; it means to what extent can Shapley value determine the level of risk. Similar to the variance contribution ratio, it can be seen from Table 2 that whether it is futures or spot, the Shapley values of short scale are very high (61.77\% and 64.18\%), indicating that the short scale determines the return risk of original returns to a certain extent. Meanwhile, the medium scale also contributes a high weight $(26.27 \%$ and $27.45 \%)$, indicating that medium scale can also determine the risk of the original data to a certain extent.

We also use the correlation coefficients between different scales and original data to identify which scale determines the overall trend change of original data. We can see from Table 3 that the correlations between original data and short scale are high (0.9268 and 0.9228). It fully shows the importance of short scale to the original data. What is interesting is the fact that the correlations between futures and spot are very high at the same scale. They are 0.5847 (short), 0.6327 (medium), and 0.7430 (long), respectively, which fully show the rationality of our multiscale hedging. On the other hand, the correlation at long scale is higher than those at other scales, mainly because the long scale eliminates the impact of short-term volatility; it can effectively highlight the consistency between futures and spot.

4.2. Hedging Results. In this subsection, we show the insample and out-of-sample hedging results of 2 hedging strategies under the minimum-CVaR framework, including the constant hedging strategy and dynamic hedging strategy. 
TABLE 3: Correlation coefficient between different scales of futures and spot.

\begin{tabular}{|c|c|c|c|c|c|c|c|c|c|}
\hline & \multirow[b]{2}{*}{ Scale } & \multicolumn{4}{|c|}{ Futures } & \multicolumn{4}{|c|}{ Spot } \\
\hline & & Original & Short & Medium & Long & Original & Short & Medium & Long \\
\hline \multirow{4}{*}{ Futures } & Original & 1.0000 & 0.9268 & 0.2879 & 0.1316 & 0.6533 & 0.5702 & 0.2405 & 0.1401 \\
\hline & Short & & 1.0000 & -0.0623 & -0.0302 & 0.5633 & 0.5847 & 0.0236 & -0.0099 \\
\hline & Medium & & & 1.0000 & 0.0443 & 0.2856 & 0.0465 & 0.6327 & 0.1222 \\
\hline & Long & & & & 1.0000 & 0.1334 & -0.0174 & 0.0170 & 0.7430 \\
\hline \multirow{4}{*}{ Spot } & Original & & & & & 1.0000 & 0.9228 & 0.2693 & 0.1447 \\
\hline & Short & & & & & & 1.0000 & -0.0683 & -0.0339 \\
\hline & Medium & & & & & & & 1.0000 & -0.0492 \\
\hline & Long & & & & & & & & 1.0000 \\
\hline
\end{tabular}

TABLE 4: In-sample minimum-CVaR hedging.

\begin{tabular}{lccccc}
\hline \multicolumn{1}{c}{ HR } & CVaR & Variance & Returns & Utility $^{\mathrm{a}}$ \\
\hline \multicolumn{7}{l}{ Panel A: constant hedge ratio } & and hedging & performance \\
Original & 0.6023 & 3.4572 & 1.3693 & 0.0009 & -10.3706 \\
Short & 0.5020 & 3.3546 & 1.4361 & -0.0197 & -10.0834 \\
Medium & 0.5231 & 1.0589 & 0.1726 & 0.0092 & -3.1674 \\
Long & 1.1938 & 0.3298 & 0.0417 & 0.0269 & -0.9625 \\
\hline Panel B: dynamic hedge ratio and hedging performance \\
Original & 0.6434 & 3.4132 & 1.3638 & 0.0083 & -10.2313 \\
Short & 0.5881 & 3.3474 & 1.4927 & 0.0335 & -10.0088 \\
Medium & 0.7792 & 0.8984 & 0.3062 & 0.2305 & -2.4648 \\
Long & 0.9056 & 0.1838 & 0.0432 & 0.1463 & -0.4050 \\
\hline
\end{tabular}

${ }^{a}$ The utility is calculated by equation $U_{p}=E\left(R_{p}\right)-0.5 A^{*} C V a R_{p}$, where $A$ is risk aversion level; by referring to Colon et al. [5], we set a moderate risk aversion level $(A=6)$.

4.2.1. In-Sample Performance. The in-sample results are estimated with the use of the entire test sample. By construction, this gives the maximum reduction in CVaR. Table 4 shows the in-sample hedging results of different hedging strategies under the minimum-CVaR framework. The $95 \% \mathrm{CVaR}$, variance, returns, and utility are selected as the criteria to provide an in-depth and comprehensive assessment of multiscale hedging strategies; we also briefly report the average estimated hedge ratios. Some findings are very attractive to us.

The optimal hedge ratios based on original returns are always based on the short-term and medium-term scales; the optimal hedge ratios based on original returns are close to those of short-term scale. These results show that the hedging performance at the short-term scale is close to using original returns, which indicates the importance of shortterm scale. Secondly, the CVaR and variance of hedged portfolio gradually decrease with the scale increasing. The variance of hedged portfolio at the long-term scale is close to 0 . There are some minor differences between static and dynamic hedging strategies. The constant hedging model performs better in variance compared to the dynamic hedging model, which indicates that the simple model may perform better. The main reason is that many parameters and assumptions of the complex models make the estimation risk greater. High estimation risk can worsen hedging performance. Thirdly, when focusing on the hedging performance from the perspective of return and utility, the overall trend of return and utility increases significantly with the scale increasing; the returns based on medium-term and long-term scales are significantly better than using the original returns, which indicate that removing short-term noise from the data can significantly improve hedging performance. Overall, the hedging performance based on long-term scale outperforms others in all respects.

Overall, our main findings are as follows. The hedging performance differs across different scales, which implies that the extracted scales comprise different information, which is useful for investors. Secondly, the hedging performance based on a short-term scale is similar to that based on original returns, implying that hedging is mainly driven by short-term information. Lastly, hedging relying on medium-term or long-term information performs better than that based on short-term information or based on all information inherent in the original returns, and hedging relying on long-term information has the best hedging performance. Therefore, excluding some information related to short-term noise from the original returns is helpful for investors.

4.2.2. Out-of-Sample Performance. The in-sample hedging performance of different hedging strategies indicates their historical performances. Since investors are concerned with how well they can hedge their positions in the future, for this motivation, we investigate the out-of-sample hedging in this subsection. The out-of-sample results are estimated every 250 days throughout the entire sample, and the estimated hedge ratios are then used to construct the hedge portfolio for the following 250 days. In detail, we reserve the first 250 observations for initial estimation of hedge ratios using equations (8) and (10), These are then used to construct the hedged portfolio for $t=251, \ldots, 500$. We then recompute the estimated hedge ratios using data for the period $t=251, \ldots, 500$ and use these to construct the hedged portfolio for $t=501, \ldots, 750$, and so on, finally resulting in 3128 out-of-sample hedge portfolio observations. Table 5 reports the average estimated hedge ratios, the 95\% CVaR, variance, returns, and utility of out-of-sample hedged portfolio. The overall results are similar to the in-sample results.

Firstly, the optimal hedge ratios at long scale are close to 1 when using dynamic hedging model, which is significantly different from the in-sample results. The out-of-sample CVaR and variance gradually decrease when the scale changes from short term to long term. The out-of-sample CVaR and 
TABle 5: Out-of-sample minimum-CVaR hedging.

\begin{tabular}{lccccc}
\hline & HR & CVaR & Variance & Returns & Utility $^{\mathrm{a}}$ \\
\hline Panel A: constant hedge ratio and hedging & performance \\
Original & 0.5999 & 3.5606 & 1.6256 & 0.0052 & -10.6767 \\
Short & 0.5201 & 3.4823 & 1.6442 & -0.0278 & -10.4748 \\
Medium & 0.5972 & 1.0861 & 0.1821 & 0.0084 & -3.2499 \\
Long & 1.1014 & 0.3245 & 0.0485 & 0.0657 & -0.9078 \\
\hline Panel B: dynamic hedge ratio and hedging performance \\
Original & 0.6433 & 3.5904 & 1.4357 & 0.0025 & -10.7687 \\
Short & 0.5984 & 3.6239 & 1.5070 & -0.0291 & -10.9009 \\
Medium & 0.7796 & 1.0846 & 0.1829 & 0.0070 & -3.2469 \\
Long & 0.9933 & 0.3425 & 0.0440 & 0.0537 & -0.9739 \\
\hline
\end{tabular}

${ }^{a}$ The utility is calculated by equation $U_{p}=E\left(R_{p}\right)-0.5 A^{*} \mathrm{CVaR}_{p}$, where $A$ is risk aversion level; by referring to Colon et al. [5], we set a moderate risk aversion level $(A=6)$.

TABLE 6: Out-of-sample minimum-variance hedging.

\begin{tabular}{lccccc}
\hline & HR & CVaR & Variance & Returns & Utility $^{\mathrm{a}}$ \\
\hline \multicolumn{5}{l}{ Panel A: constant hedge ratio and hedging } & performance \\
Original & 0.6372 & 3.6024 & 1.4443 & 0.0016 & -4.3314 \\
Short & 0.5638 & 3.5192 & 1.4948 & -0.0265 & -4.5107 \\
Medium & 0.6422 & 1.0791 & 0.1782 & 0.0081 & -0.5264 \\
Long & 1.0155 & 0.4072 & 0.0547 & 0.0755 & -0.0887 \\
\hline Panel B: dynamic hedge ratio and hedging & performance \\
Original & 0.6517 & 3.6082 & 1.4424 & 0.0030 & -4.3243 \\
Short & 0.5826 & 3.5481 & 1.4937 & -0.0279 & -4.5089 \\
Medium & 0.7472 & 1.0958 & 0.1819 & 0.0071 & -0.5385 \\
Long & 0.9018 & 0.3619 & 0.0457 & 0.0437 & -0.0934 \\
\hline
\end{tabular}

${ }^{a}$ The utility is calculated by equation $U_{p}=E\left(R_{p}\right)-0.5 A^{*} \operatorname{var}_{p}$, where $A$ is risk aversion level; by referring to Colon et al. [5], we set a moderate risk aversion level $(A=6)$.

variance based on short-term scale and original returns are very similar, reconfirming the point that hedging is mainly driven by short-term information. Overall, the hedging performance based on original returns performs better than using short-term information, due to the fact that the original returns also contain medium-term and long-term information, which is critical to improving hedge performance. Lastly, the overall trends of return and utility increase significantly with the scale increasing; the returns based on medium-term and long-term scales are significantly better using the original returns. These findings are consistent with the out-of-sample $\mathrm{CVaR}$ and variance indicators, reconfirming the in-sample conclusion that removing the short-term noise from the original returns is useful for investors.

\subsubsection{Hedging Performance under the Minimum-Variance} Framework. To check the robustness of our findings, we also show the out-of-sample results under the minimum-variance framework. Table 6 presents the relevant results. For space limitation, we only report the out-of-sample results. The hedging results under the minimum-variance framework are consistent with minimum-CVaR hedging on the whole trend, while there is a slight difference. When the scale changes from short term to long term, the trends of CVaR, variance, returns, and utility are consistent with the
minimum-CVaR hedging results, which show that our conclusions are robust and universal. Besides, the minimum-variance hedging performance is worse than the minimum-CVaR hedging strategy in reducing the risk of hedged portfolio. For example, when we use dynamic hedging strategy to hedge the spot risk in original returns, the CVaR and variance of the hedged portfolio are 3.6082 and 1.4424 under the minimum-variance framework, while the results are 3.5904 and 1.4357 under the minimum-CVaR framework. Undoubtedly, the minimum-CVaR hedging strategy is a better choice for investors, since the investors' main objective in hedging is to reduce the spot risk of crude oil, especially the downside tail risk.

\section{Conclusions}

In this study, we use EMD technology to decompose crude oil returns and study the effect of different time and frequency scales on hedging. In detail, the EMD technology is first introduced to decompose crude oil futures and spot data and reconstruct the decomposition terms into three different scales: short-term, medium-term, and long-term. Then, we discuss the scale dependency structure through three key indicators: variance contribution rate, Shapley value, and correlation coefficient. Lastly, under the minimum-CVaR framework, two different hedging strategies, constant hedging strategy and dynamic hedging strategy, using the DCC-GARCH model, are selected to comprehensively analyze the crude oil hedging performance at different scales.

In the empirical analysis, the daily closing prices of spot and futures contracts of rent crude oil were from August 18, 2005, to September 16, 2019, and a total of 3379 price observations are collected to test in-sample and out-of-sample hedging effectiveness. We select the CVaR, variance, returns, and utility as the criteria to provide an in-depth and comprehensive assessment of the hedging performance at different scales. The empirical results show that the extracted scales comprise different information on original returns, Secondly, short-term information occupies the most important position, and hedging is mainly driven by short-term information. Lastly, hedging relying on long-term information has the best hedging performance; excluding some information related to short-term noise from the original returns is helpful for investors. Robustness results by changing the objective function to variance validate the above conclusions.

\section{Data Availability}

The datasets used in this paper are available from the website of the Intercontinental Exchange (ICE) (www.theice.com).

\section{Conflicts of Interest}

The authors declare that they have no conflicts of interest.

\section{Acknowledgments}

This research was supported by Humanities and Social Science Planning Fund Project of the Ministry of Education 
(16YJAZH078) and Central University for Basic Research Business Expenses (CCNU19TS062).

\section{References}

[1] Y. Wang, Z. Pan, L. Liu, and C. Wu, "Oil price increases and the predictaility of equity premium," Journal of Anking \& Finance, vol. 102, pp. 43-58, 2019.

[2] D. Zhang, Q. Ji, and A. M. Kutan, "Dynamic transmission mechanisms in gloal crude oil prices: estimation and implications," Energy, vol. 175, pp. 1181-1193, 2019.

[3] A. David, "Exploration activity, long-run decisions, and the risk premium in energy futures," The Review of Financial Studies, vol. 32, no. 4, pp. 1536-1572, 2019.

[4] Y. Wang, Q. Geng, and F. Meng, "Futures hedging in crude oil markets: a comparison etween minimum-variance and minimum-risk frameworks," Energy, vol. 181, pp. 815-826, 2019.

[5] T. Conlon, J. Cotter, and R. Gençay, "Commodity futures hedging, risk aversion and the hedging horizon," The European Journal of Finance, vol. 22, no. 15, pp. 1534-1560, 2016.

[6] S.-S. Chen, C.-F. Lee, and K. Shrestha, "Futures hedge ratios: a review," Encyclopedia of Finance, vol. 43, no. 3, pp. 433-456, 2010.

[7] W. Opie and S. J. Riddiough, "Gloal currency hedging with common risk factors," Journal of Financial Economics, vol. 136, no. 3, pp. 780-805, 2020.

[8] L. H. Ederington, "The hedging performance of the new futures markets," The Journal of Finance, vol. 34, no. 1, pp. 157-170, 1979.

[9] M. Billio, R. Casarin, and A. Osuntuyi, "Markov switching GARCH models for ayesian hedging on energy futures markets," Energy Economics, vol. 70, pp. 545-562, 2018.

[10] J. Wang, M. C. Zhou, X. Jin et al., "Variance minimization hedging analysis based on a time-varying Markovian DCCGARCH model," IEEE Transactions on Automation Science and Engineering, vol. 17, no. 2, pp. 621-632, 2019.

[11] D. I. Okorie and B. Lin, "Crude oil price and cryptocurrencies: evidence of volatility connectedness and hedging strategy," Energy Economics, vol. 87, Article ID 104703, 2020.

[12] S. Chai and P. Zhou, "The Minimum-CVaR strategy with semi-parametric estimation in caron market hedging prolems," Energy Economics, vol. 76, pp. 64-75, 2018.

[13] P. Artzner, F. Delaen, J.-M. Eer, and D. Heath, "Coherent measures of risk," Mathematical Finance, vol. 9, no. 3, pp. 203-228, 1999.

[14] R. T. Rockafellar and S. Uryasev, "Conditional value-at-risk for general loss distriutions," Journal of Anking \& Finance, vol. 26, no. 7, pp. 1443-1471, 2002.

[15] D. Chun, H. Cho, and J. Kim, "Crude oil price shocks and hedging performance: a comparison of volatility models," Energy Economics, vol. 81, pp. 1132-1147, 2019.

[16] T. Conlon and J. Cotter, "An empirical analysis of dynamic multiscale hedging using wavelet decomposition," Journal of Futures Markets, vol. 32, no. 3, pp. 272-299, 2012.

[17] J. Sultan, A. K. Alexandridis, M. Hasan, and X. Guo, "Hedging performance of multiscale hedge ratios," Journal of Futures Markets, vol. 39, no. 12, pp. 1613-1632, 2019.

[18] J. Bi, H. Yuan, and M. Zhou, "Temporal prediction of multiapplication consolidated workloads in distriuted clouds," IEEE Transactions on Automation Science and Engineering, vol. 16, no. 4, pp. 1763-1773, 2019.

[19] N. E. Huang, Z. Shen, S. R. Long et al., "The empirical mode decomposition and the Hilert spectrum for nonlinear and non-stationary time series analysis," Proceedings of the Royal Society of London. Series A: Mathematical, Physical and Engineering Sciences, vol. 454, no. 1971, pp. 903-995, 1998.

[20] C. Liu, X. Sun, J. Wang, J. Li, and J. Chen, "Multiscale information transmission etween commodity markets: an EMD-ased transfer entropy network," Research in International Usiness and Finance, vol. 55, Article ID 101318, 2021.

[21] B. Zhu, D. Han, P. Wang, Z. Wu, T. Zhang, and Y-M. Wei, "Forecasting caron price using empirical mode decomposition and evolutionary least squares support vector regression," Applied Energy, vol. 191, pp. 521-530, 2017.

[22] B. Wang and J. Wang, "Energy futures and spots prices forecasting y hyrid SW-GRU with EMD and error evaluation," Energy Economics, vol. 90, Article ID 104827, 2020.

[23] Z. Cao, R. D. Harris, and J. Shen, "Hedging and value at risk: a semi-parametric approach," Journal of Futures Markets, vol. 30, no. 8, pp. 780-794, 2010.

[24] R. Engle, "Dynamic conditional correlation," Journal of Usiness \& Economic Statistics, vol. 20, no. 3, pp. 339-350, 2002.

[25] L. Yang, L. Zhao, and C. Wang, "Portfolio optimization ased on empirical mode decomposition," Physica A: Statistical Mechanics and Its Applications, vol. 531, Article ID 121813, 2019.

[26] E. Winter, "Chapter 53 the shapley value," Handook of Game Theory with Economic Applications, vol. 3, pp. 2025-2054, 2002. 